\title{
Properties of the linearly polarized radiation from PSR B0950+08
}

\author{
T. V. Shabanova and Yu. P. Shitov
}

\author{
Astro Space Center, P. N. Lebedev Physical Institute, Leninskij Prospect 53, 117924 Moscow, Russia \\ e-mail: shitov@prao.psn.ru
}

Received 30 July 2002 / Accepted 26 November 2003

\begin{abstract}
Measurements of average pulse profiles made with a single linear polarization over the range 41-112 $\mathrm{MHz}$ are presented for PSR B0950+08. We show that the observed variable structure of the pulse profiles is a result of Faraday sinusoidal modulation of the pulse intensity with frequency. The rotation measure corresponding to this effect, $R M \approx 4 \mathrm{rad} \mathrm{m}^{-2}$, is about 3 times greater than the published value of $R M=1.35 \mathrm{rad} \mathrm{m}^{-2}$ (Taylor et al. 1993).
\end{abstract}

Key words. stars: neutron - stars: pulsars: general - stars: pulsars: individual: PSR B0950+08

\section{Introduction}

PSR B0950+08 is well-studied over a wide frequency range from 24 to $10500 \mathrm{MHz}$. It exhibits a single pulse profile at frequencies above $400 \mathrm{MHz}$ and a double pulse profile at low frequencies in the range 24-112 MHz (Hankins et al. 1991; Kuzmin et al. 1998). There is an interpulse occurring approximately $152^{\circ}$ ahead of the main pulse and a bridge of emission between the interpulse and main pulse (Lyne \& Rickett 1968).

The study of average pulse profiles from a large number of pulsars has shown that a pulse profile obtained by averaging several hundred individual pulses is very stable for most pulsars (Helfand et al. 1975; Rathnasree \& Rankin 1995). Profile changes can be caused by such phenomena as mode changing and nulling which occur in a small fraction of pulsars. The narrowband profile changes which are observed for the pulsar B0950+08 in the range 41-112 MHz and discussed in this paper are consistent with the effects of Faraday rotation on a linearly polarized pulse emission received by a linearly polarized antenna. Pulsars with considerable linear polarization observed with a single linear polarization show frequencydependent profiles because of Faraday rotation of the plane of polarization in the interstellar medium. Pulse profiles obtained for different epochs show time-dependent profile shapes caused by a variation of the electron density or magnetic field in the propagation path, mainly due to varying ionospheric contribution to the rotation measure. At the Pushchino Radio Astronomy Observatory (PRAO), the effect of Faraday modulation of the pulse intensity with frequency at the output of the multi-channel receiver is used for the measurements of linear polarization characteristics of pulsars and the estimation of their rotation measure (Vitkevich \& Shitov 1970; Shitov 1971; Suleymanova 1989).

Send offprint requests to: T. V. Shabanova, e-mail: tvsh@prao.psn.ru
Changes of the average pulse profile at meter wavelengths for the pulsar B0950+08 were first noticed by Smirnova \& Shabanova (1992). They found that the pulse profile is variable in time and shows narrowband changes with frequency. The explanation of the observed phenomenon by Faraday rotation was problematic because the predicted effect of the interstellar Faraday rotation for the tabulated value of $R M=$ $1.35 \mathrm{rad} \mathrm{m}^{-2}$ (Taylor et al. 1993) was negligible across the receiver bandpass at $102 \mathrm{MHz}$. The authors supposed that the narrowband changes of the pulse profile may be due either to the pulsar's intrinsic narrowband emission, manifesting only at low frequencies, or to scintillations of spatially separate sources. However, the low time resolution of their observations (about $10^{\circ}$ of longitude) made a detailed analysis of this phenomenon impracticable.

The main purpose of this paper is to explain the observed narrowband profile changes of PSR B0950+08. The techniques used for observations over the frequency range $41-112 \mathrm{MHz}$ are described in Sect. 2. In Sect. 3 a large set of average profiles is investigated with respect to the shape changes with time. In Sect. 4 we study the narrowband changes of the pulse profile across the $2.56-\mathrm{MHz}$ bandpass and establish a relation between the shape changes with time and the shape changes with frequency. Section 5 discusses the frequency dependence of the pulse profile after removing the effect caused by interstellar scintillation. In Sect. 6 we compute Faraday rotation effects using a numerical polarization model of the pulse profile. Section 7 describes the narrowband changes of the average profile at the lower frequencies of 88,62 and $41 \mathrm{MHz}$. Section 8 presents the results of the timing data analysis for the pulsar. In Sect. 9 we conclude that the profile changes are due to the Faraday rotation effect.

\section{Observations}

Observations with a high time resolution of average pulse profiles and individual pulses from the pulsar B0950+08 were 
carried out at PRAO in three observing sessions from April 1996 to May 2002.

The first session took place from April 1996 through July 2001 and included regular timing observations of the pulsar with the BSA radiotelescope which operated at $102.7 \mathrm{MHz}$ until May 1998, and at 111.3 MHz since November 1998. The BSA large phased array radiotelescope, making up a linearly polarized transit antenna with $30000 \mathrm{~m}^{2}$ effective area and a beam size of about $(3.5 / \cos \delta)$ min, provides 3.3 min duration observations at the pulsar declination $\delta \approx 8^{\circ}$. A 32 channel $\times$ $5 \mathrm{kHz}$ spectrometer covering a total bandwidth of $160 \mathrm{kHz}$ was used to provide high frequency resolution. The receiver time constant was $0.3 \mathrm{~ms}$. The data were sampled at intervals of $0.2048 \mathrm{~ms}$. An average pulse profile in each channel was formed by synchronous averaging of 770 individual periods with a predicted topocentric pulsar period. After dispersion removal all the channel profiles were summed to form an averaged pulse profile for a single observation. A narrow bandwidth of one $5-\mathrm{kHz}$ channel limited the pulse broadening from the interstellar dispersion to $0.09 \mathrm{~ms}$. The observation window width was $200^{\circ}$ of longitude. A total of 264 average pulse profiles were derived at two observing frequencies around 102.5 and $112 \mathrm{MHz}$ for a 5-yr span of observations.

The second session included observations of individual pulses and was made using the BSA telescope at an observing frequency of $111.87 \mathrm{MHz}$ during 2001 and 2002. The $128 \mathrm{spec}-$ tral channels, each of bandwidth $20 \mathrm{kHz}$, were used to record individual pulses for $3.3 \mathrm{~min}$ of the BSA transit time. The receiver time constant was $1 \mathrm{~ms}$ and the sampling interval was 0.512 or $0.8192 \mathrm{~ms}$. The signal dispersion through one $20-\mathrm{kHz}$ channel causes pulse broadening of $0.35 \mathrm{~ms}$.

The third session was carried out using the East-West arm of the DKR-1000 radiotelescope at three lower frequencies of $88.57,62.15$ and $41.07 \mathrm{MHz}$ in late 2001 and early 2002. Individual pulses were recorded during $16 \mathrm{~min}$ of the DKR transit observation time with the 128 channel $\times 20 \mathrm{kHz}$ spectral radiometer. The parameters of the DKR observations are listed in Table 1. The effects of the interstellar dispersion in the total bandwidth of $2.56 \mathrm{MHz}$ at three observing frequencies were 91, 263 and $911 \mathrm{~ms}$, respectively. The dispersion time delay at 62.15 and $41.07 \mathrm{MHz}$ was more than the 253-ms pulsar period. To compensate for this time delay, the signal was recorded blocks of 5 full periods at $62.15 \mathrm{MHz}$ and blocks of 12 full periods at $41.07 \mathrm{MHz}$. From each block, the signal was only analyzed for either the 3 or 8 first successive periods at each observing frequency, respectively. The average profile was formed by addition of 2200 pulse periods at $62.15 \mathrm{MHz}$ and 2160 pulse periods at $41.07 \mathrm{MHz}$.

The pulsar B0950+08 shows a high degree of linear polarization of about $90 \%$ and a smooth change in the position angle through the interpulse and main pulse, as measured at 150 and $151 \mathrm{MHz}$ (Schwarz \& Morris 1971; Lyne et al. 1971). Both the BSA and DKR radiotelescopes receive only one polarization. For our observations, the published value of a rotation measure of $R M=1.35 \mathrm{rad} \mathrm{m}^{-2}$ (Taylor et al. 1993) predicts negligible effect of Faraday rotation across the $2.56-\mathrm{MHz}$ bandpass. The expected periods of Faraday modulation of the pulsar emission with frequency are $18 \mathrm{MHz}$ at the observing
Table 1. Parameters of the DKR observations.

\begin{tabular}{llll}
\hline \hline Parameter & Value & & \\
\hline Observing frequency (MHz) & 88.57 & 62.15 & 41.07 \\
Receiver time constant (ms) & 1 & 1 & 3 \\
Sampling interval (ms) & 0.8192 & 0.8192 & 2.9696 \\
One bandwidth (MHz) & 0.02 & 0.02 & 0.02 \\
Dispersion pulse broadening (ms) & 0.7 & 2 & 7 \\
Total bandwidth (MHz) & 2.56 & 2.56 & 2.56 \\
Dispersion time delay (ms) & 91 & 263 & 911 \\
Number of pulses averaged & 3770 & 2200 & 2160 \\
\hline
\end{tabular}

frequency of $112 \mathrm{MHz}, 14 \mathrm{MHz}$ at $102.7 \mathrm{MHz}, 9 \mathrm{MHz}$ at 88.6 MHz, $3 \mathrm{MHz}$ at $62 \mathrm{MHz}$ and $0.9 \mathrm{MHz}$ at $41 \mathrm{MHz}$. This effect produces a modulation of the pulse emission spectrum of less than $15 \%$ at the frequencies within the $102-112 \mathrm{MHz}$ range and is significant only at two lower frequencies of 62 and $41 \mathrm{MHz}$. The influence of a varying ionosphere on our measurements for different days is also small. Most observations of the pulsar were made at night time or close to it (during autumn or winter), when an ionospheric contribution to the rotation measure $R M$ is estimated to be less than $0.4 \mathrm{rad} \mathrm{m}^{-2}$ for the PRAO site (Udaltsov \& Zlobin 1974).

\section{The changes of the average profile with time in the range 102-112 $\mathrm{MHz}$}

We investigated average profiles using timing observations over the interval 1996-2001. The shape of the observed pulse profiles strongly varies from one observation to another that we can see both double and triple profiles. To reveal some regularities in the occurrence of the profiles with different shapes, we sorted available average profiles into groups, each containing similar profile shapes. Each of the 264 observed profiles could be put into one of the profile groups. A detailed analysis of these data showed that the shapes from different groups make up a set smoothly varying between the main states of the double profile. Such a set of continuously varying shapes is presented in Fig. 1.

The shape (a) represents the main pulse, which has two well-resolved components with a separation of $10^{\circ}$. The shapes from (b) to (g) show the well-resolved third component, occurring $34^{\circ}$ ahead of the main pulse and visible at a longitude of about $170^{\circ}$. Note that the unresolved component ahead of the main pulse in the range $151-430 \mathrm{MHz}$ was observed earlier (Lyne et al. 1971; Hankins \& Cordes 1981). The shape changes affect the whole profile and both the components of the main pulse alter their amplitude, either amplifying or weakening alternately. Variations in the relative amplitudes of all three components exhibit a periodic character.

The two profiles (c) and (d) of Fig. 1 have a lower signal-to-noise ratio $(\mathrm{S} / \mathrm{N})$ and show unusual shapes. These profiles are similar to the adjacent profiles (b) and (f) having a better $\mathrm{S} / \mathrm{N}$ ratio. Shapes (c) and (d) are of great importance to confirm the periodic character of changes in the pulse profile. These shapes provide a transition between the first and second 


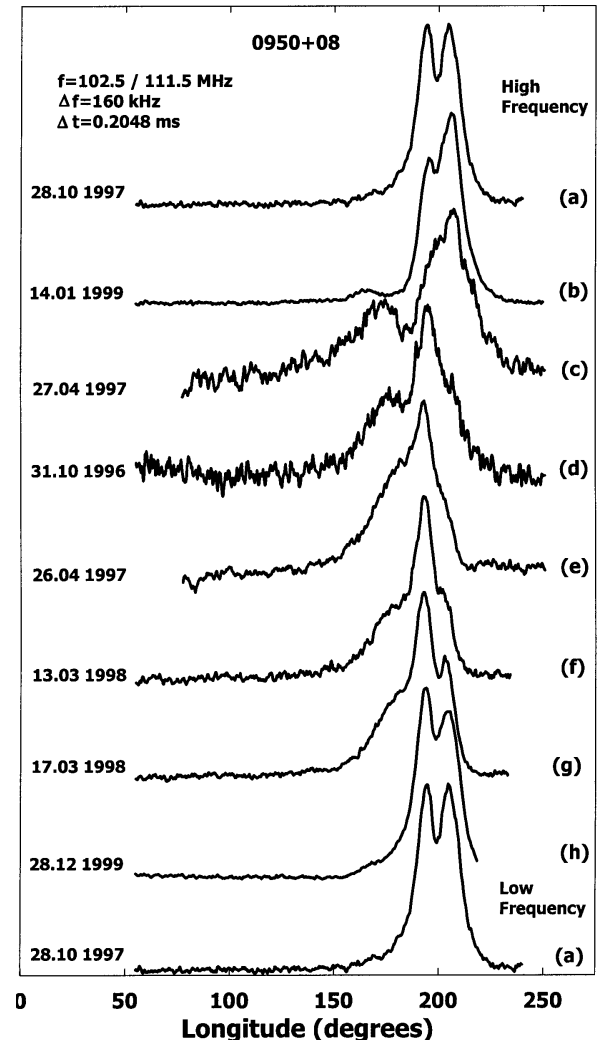

Fig. 1. A sequence of shapes of the average profile continuously varying between the main states of the double profile. The profiles were chosen from the full data set obtained between 1996 and 2001 at frequencies around 102 and $112 \mathrm{MHz}$. Shapes from $\mathbf{b}$ ) to $\mathbf{g}$ ) show a triple profile with a well-resolved component ahead of the main pulse located at longitudes from $150^{\circ}$ to $180^{\circ}$. The sample interval is 0.3 of longitude. All the profiles are aligned in time and normalized to unity.

dominant components of the main pulse. The state (c) where the peak of the second component is significantly higher than the peak of the first one quickly changes to another state (d) where the peak of the first component dominates.

\section{Changes of pulse shapes with frequency}

Individual pulses from the pulsar were observed at $111.87 \mathrm{MHz}$ with the 128 channel $\times 20 \mathrm{kHz}$ spectral radiometer. The high quality data set was analyzed to investigate the frequency structure of the average profiles within bands of different widths of 640,160 and $20 \mathrm{kHz}$.

The pulse profiles were obtained by averaging 770 individual periods for each 32-channel quadrant of the filter bank. The sequences of 4 resulting profiles are presented in Fig. 2 for three different observations. Detailed inspection of each panel shows that the shape of the average profile changes markedly over the bandpass of $2.56 \mathrm{MHz}$. It is also seen that the sequences shown are not identical although the same shapes occur in all the cases. An analysis of the available data showed that the profile shapes vary with frequency in a strictly defined order and that the frequency changes are a result of some modulation process having a phase drift with time. If the process observed is related to Faraday rotation then the tabulated value of $R M$ would be expected to be wrong. Therefore the predicted Faraday effect cannot be negligible. Variable ionospheric Faraday rotation does not affect the profiles during the 3 min of observation time but causes a drift of the frequency picture with time.

The receiver bandwidth is less than the frequency interval of the modulation process and so we observe only some stages of this process on different days. Nevertheless, a period of the profile frequency changes can be estimated if we analyze the records together, which contain overlapping shapes as shown in Fig. 2. Here the last shape at $109.95 \mathrm{MHz}$ of the panel (a) is similar to the first shape at $111.87 \mathrm{MHz}$ of panel (b). This is also the case for panels (b) and (c). A set of 12 plotted profiles covers the full cycle of the shape changes and shows how the frequency picture develops from higher to lower frequencies, regardless of the particular value of the observing frequency. The transition between the first and second dominant components, which was discussed in the preceding section, is clearly observed in panel (a) between the shapes at 111.23 and $110.59 \mathrm{MHz}$ and panel (c) between the shapes at 111.87 and $111.23 \mathrm{MHz}$. This state indicates a transition to a new cycle of profile changes with frequency. The interval between these states is $6-7640-\mathrm{kHz}$ bands, i.e. it is approximately equal to $4 \mathrm{MHz}$. Hence, having a 2.56-MHz band, we can nevertheless estimate the frequency interval of the shape changes which can be several times wider than a bandwidth.

For a more detailed analysis of the profile frequency structure, individual pulses were averaged within narrower $160-\mathrm{kHz}$ bands. Two sets of 16 resulting profiles are presented in Fig. 3 for two different epochs. They give a finer frequency structure of the $640-\mathrm{kHz}$ profiles, already displayed in Figs. 2a and b. In order to see the profile frequency dependence inside one cycle of changes, the right hand sequence should be considered as an extension of the left hand one. The first component of the profile at $111.87 \mathrm{MHz}$ (panel (a)) weakens gradually toward lower frequencies and becomes unresolved at $110.59 \mathrm{MHz}$. Beginning from the profile at $110.43 \mathrm{MHz}$, the first component dominates. The last 4 profiles from 109.95 to $109.47 \mathrm{MHz}$ of this sequence are similar to the first 4 profiles of the right hand sequence. The second component becomes visible in the profile at $111.23 \mathrm{MHz}$ (panel (b)). At $110.27 \mathrm{MHz}$, the two components become comparable in amplitude. Then, the first component becomes again weaker and the picture is repeated. The profile at $109.79 \mathrm{MHz}$ of the panel (b) is similar to the first profile at $111.87 \mathrm{MHz}$ of the panel (a). It should be noted that the left and right sequences show different rates of shape changes with frequency. It may be caused by a varying ionospheric contribution to the rotation measure on different days.

We see that the set of the profile shapes plotted in Fig. 1 is similar to the set of the shapes presented in Fig. 3. This suggests that the profile changes with time are a result of the profile changes with frequency. When the receiver bandpass is narrower than the frequency interval of the shape changes, the profile shape obtained will depend on the location of the band inside this interval. Note that the pulse profiles of Fig. 1 were plotted in such a way that the sequence of the profile shapes placed from top to bottom would correspond to the sequence 


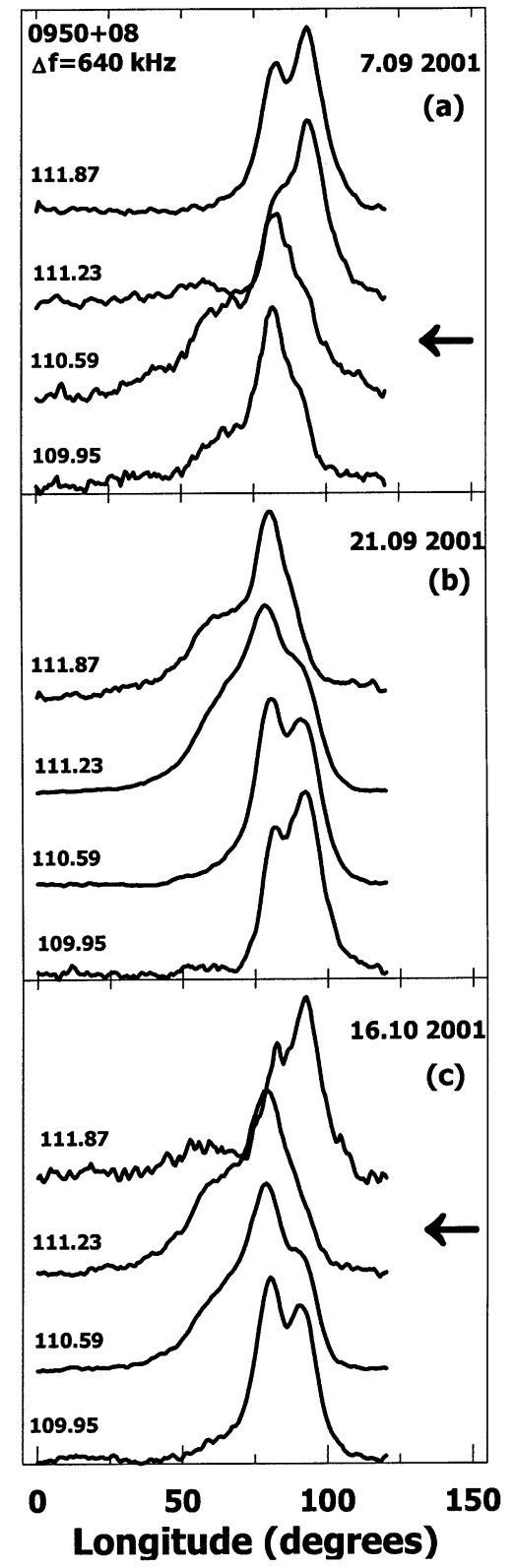

Fig. 2. The changes of the average profile with frequency for three different epochs. A set of 12 plotted profiles covers the full cycle of the shape changes. Panels a) and c) demonstrate the transition state between the first and second dominant components of the main pulse (marked by arrow). The sample interval is 0.7 of longitude. All the profiles are normalized to unity.

of the profile shapes observed within the receiver bandpass in the direction from a higher frequency to a lower one.

An analysis showed that the shape changes are clearly seen even in individual channels of $20-\mathrm{kHz}$ width and these changes cannot be caused by instrumental effects. The transition state may occupy a bandwidth from 40 to $160 \mathrm{kHz}$ for different observations. Weakening of the signal is a typical feature for all the observations where this state occurs.
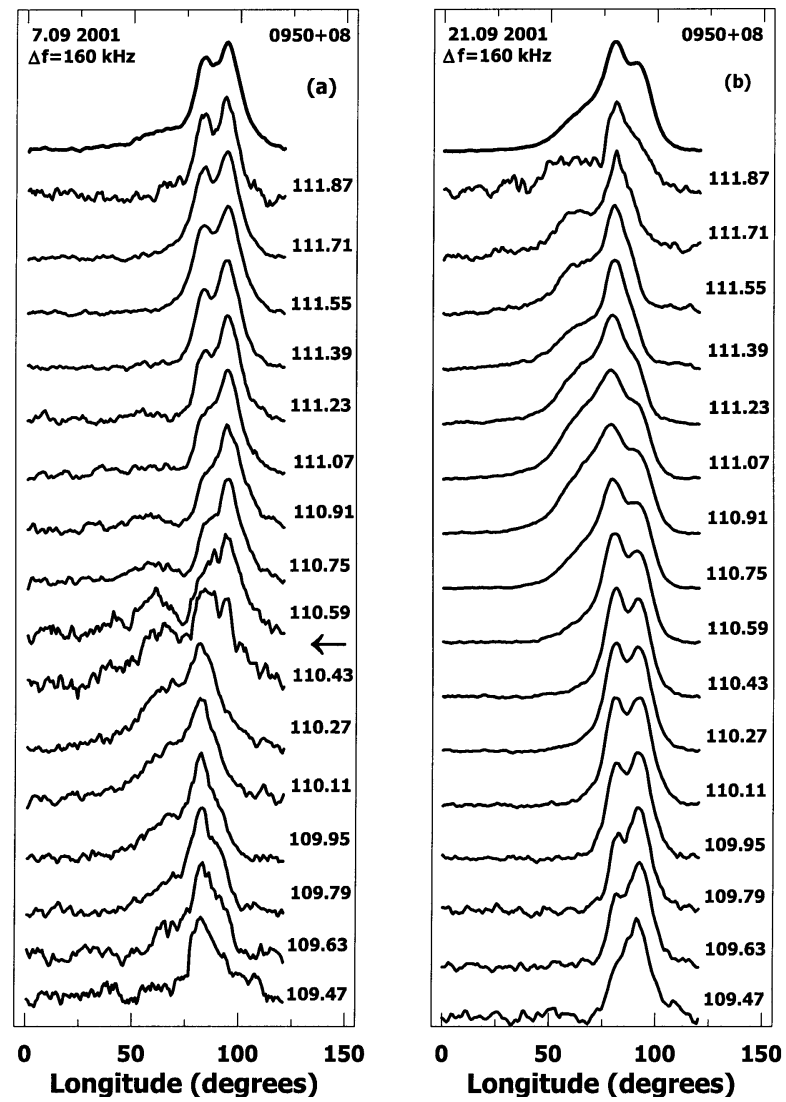

Fig. 3. The frequency changes of the average profile between $160-\mathrm{kHz}$ bands for two different epochs. To watch the profile frequency dependence inside one cycle of changes, the right hand sequence should be considered as an extension of the left hand one. The transition state between the first and second dominant components is marked by the arrow. All the profiles are normalized to unity.

\section{Interstellar scintillations and the frequency structure of the profile changes at $112 \mathrm{MHz}$}

It is well known that the pulse emission of PSR B0950+08 is modulated by interstellar scintillations. In order to determine the type of frequency dependence of the profile changes we must exclude modulation due to scintillations. The emission spectrum of the pulsar within the receiver bandpass was obtained using the following procedure. For each $20-\mathrm{kHz}$ channel out of 128, the area under the average profile was calculated on the longitude interval above the 0.1 level of the profile peak and normalized to the rms of noise outside the profile in the same channel. The spectrum of scintillations is characterized by modulation of the area under the average profile with frequency and is estimated by the width of an autocorrelation function $A C F$. For our observations at $112 \mathrm{MHz}$, modulation of the emission spectrum of the pulsar is close to $80 \%$. The width of the decorrelation band of the spectrum of scintillations defined by the half-width at the $(1 / e)$ level of $A C F$ is equal to $250 \mathrm{kHz}$. This agrees well with earlier measurements at $105 \mathrm{MHz}$ made by Shitov (1972).

The emission spectrum of the pulsar in the receiver bandpass was calculated by dividing the amplitude of the average profile for each channel by the area under the average profile in 
this channel. Some examples of the observed emission spectra at the longitudes of pulse peaks of the three components are given in the left panel of Fig. 4 for different observations.

As is seen in this plot, the spectra of the two main components of the pulse profile intersect at two points, which correspond to two basic shapes of the average profile. Point 1 corresponds to a transition state between the first and the second dominant components of the main pulse (see triple profiles (c) and (d) in Fig. 1). Here the pulse peak of the first component becomes higher than the pulse peak of the second component. Near this point, the pulse peak of the third component ahead of the main pulse has the greatest value. Point 2 corresponds to a state when the pulse peaks of both the dominant components have comparable amplitudes, and the amplitude of the third component approaches its smallest value (see double profile (a) in Fig. 1).

Uniform behavior of the spectral curves for all the observations can specify that the narrowband changes in the pulse shape are deterministic. A detailed analysis of these data showed that the frequency structure of the pulse profile can be modelled by a sinusoid. We approximated the obtained spectra of the pulse emission by three sinusoidal curves, defined for each component as

$y 1(k)=0.20 \sin \left(\frac{2 \pi}{T} * k+\phi\right)+0.60$,

$y 2(k)=0.25 \sin \left(\frac{2 \pi}{T} * k-0.4+\phi\right)+0.60$,

$y 3(k)=0.19 \sin \left(\frac{2 \pi}{T} * k+\pi+\phi\right)+0.19$,

where $T$ is the period of the sinusoidal curve measured in the channels, $\phi$ is a variable phase of the sinusoidal curve and $k$ is the channel number. The amplitudes of the three approximation curves and their constant phase shifts were defined by a method of successive approximations. A necessary condition was that the cross points of the three sinusoids should correspond to the cross points of the observed curves. It was assumed that the approximation curve $y(k)$ gives a good fit when the goodnessof-fit parameter, $S=\sum_{k=1}^{128}[f(k)-y(k)]^{2}$, is minimum. Here $f(k)$ is the observed curve. A formal least-squares fitting procedure was not applied because it gave unreasonable estimations of the parameters in view of the large scattering of the experimental data and short segments of the observed curves.

We applied the approximation procedure to all the cases presented in the left panel of Fig. 4, varying the frequency scale $T$ and location $\phi$ of the $2.56-\mathrm{MHz}$ band. Initial amplitudes and phases of the curves remained the same. The results are presented in the right panel of Fig. 4 in the same scale. Comparison of the spectra plotted in both panels indicates that the suggested model approximates the observed cases very well. The profile changes at the longitudes of the three components show appreciable sinusoidal dependence on frequency. The frequency scale of the shape changes varies from 140 to 450 channels (or from 3 to $9 \mathrm{MHz}$ ) for different observations at $112 \mathrm{MHz}$.
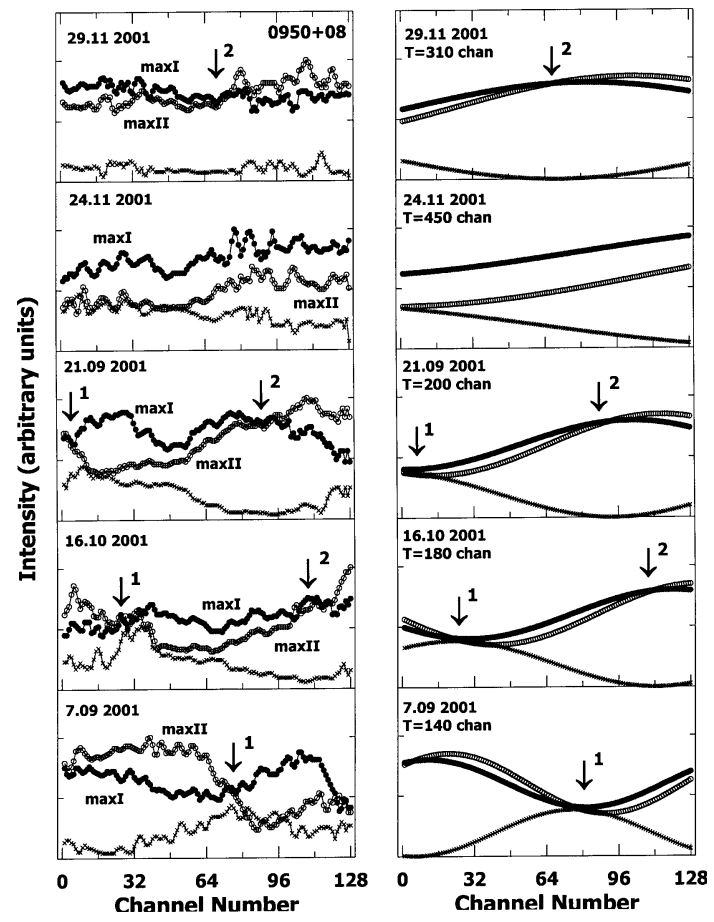

Fig. 4. The frequency structure of the average profile at the longitudes of the pulse peaks of the three components for different epochs: the observed spectra (left), the model spectra (right). The spectrum for the first component of the main pulse is marked by filled circles and the word "maxI", the spectrum for the second component is marked by open circles and the word "maxII", the spectrum for the third component is marked by crosses.

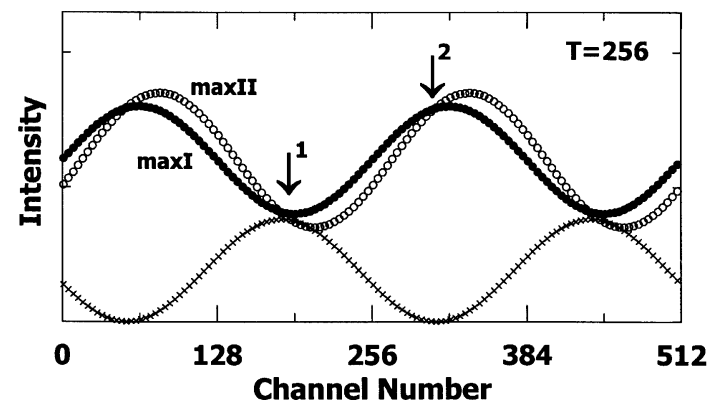

Fig. 5. The model picture of the amplitude changes of the average profile with frequency at the longitudes of the pulse peaks of the three components for $T=256$ and $\phi=0$. The spectrum is marked by filled circles and the word "maxI" for the first component, open circles and the word "maxII" for the second component and crosses for the third one.

A more detailed picture of the amplitude changes of the average profile with frequency is shown in Fig. 5. The approximation curves were calculated for $T=256$ and $\phi=0$. A sinusoidal modulation covers all the three components of the average profile, but at the longitudes of each component it happens with different phase lags. The lags do not depend on time. This produces the profile shapes varying with frequency in a similar way for different observations. Figure 5 also shows that all the observed profile shapes can be explained by a different location of the receiver bandpass within a cycle of the sinusoidal curve. 


\section{Numerical polarization model of the average profile}

The frequency picture shown in Fig. 5 is very similar to a Faraday rotation pattern. To clarify whether the observed effect is due to Faraday rotation, we determined a numerical polarization model of the profile and computed the effects due to Faraday rotation using the technique that is applied for measurements of linear polarization characteristics of pulsars at PRAO. The frequency-time dependence of a Faraday rotation pattern was computed using the equation

$A_{k}(i)=a(i) \sin \left[\frac{2 \pi}{T} * k+2 \phi(i)\right]+b(i)$

where $k$ is the channel number, $i$ is the pulse longitude, $T$ is a period of a sinusoidal curve measured in channels, $\phi(i)$ is a position angle profile, and $a(i)$ is an amplitude of sinusoidal modulation of the polarized component. The computed values of $A_{k}(i)$ give the Faraday rotation spectrum of the average profile and corresponding pulse shapes in each channel. The results are presented in Fig. 6.

In computing $A_{k}(i)$ we used the published polarization profiles of PSR B0950+08 at low frequencies at 150 and $151 \mathrm{MHz}$ given in Schwarz \& Morris (1971) and Lyne et al. (1971). The model profile adopted is presented in Fig. 6 a that shows the average profile in total intensity $I(i)$, the linearly polarized component $P(i)$, and the position angle of polarization $\phi(i)$. The profile for $I(i)$ is normalized to 100 at maximum. In this model, the unresolved component ahead of the main pulse is $100 \%$ linearly polarized (longitude interval from $1^{\circ}$ to $29^{\circ}$ ). The degree of linear polarization, $P_{\mathrm{L}}$, for the first and second components of the main pulse is $75 \%$ and $80 \%$, respectively. Their corresponding longitude intervals are $29^{\circ}-44^{\circ}$ and $44^{\circ}-66^{\circ}$.

The amplitude of sinusoidal curve $a(i)$ in Eq. (4) was defined by the relation $a(i)=P(i) / 2$, where $P(i)=I(i) * P_{\mathrm{L}}(i)$ is the intensity of the polarized component at longitude $i$. The mean level of the pulse intensity $b(i)$ at longitude $i$ was found from $b(i)=I(i) / 2$. The period of the sinusoidal curve was assumed equal to $T=256$ channels, the same as in Fig. 5. According to Lyne et al. (1971), the position angle $\phi$ swings $160^{\circ}$ through the profile. This gives the phase offset between the Faraday spectra of two components of the main pulse of about -0.4 radian, i.e. the same as in Eq. (2). To obtain the phase offset between the spectra of the first and third components equal to $\approx \pi$, as specified in Eq. (3) and Fig. 5, we should suppose that the position angles for these components are orthogonal. The finally adopted behavior of the position angle is given in Fig. $6 \mathrm{a}$ in the top panel. Here the position angle swings $200^{\circ}$ through the profile and decreases across the profile with a higher rate of decrease in the range of the third component. This gives about $90^{\circ}$ displacement from the position angle of the first component.

The resulting frequency dependence of the pulse profile at the longitudes of the pulse peaks of the three components is given in Fig. 6b. Comparison with Fig. 5 shows that the computed Faraday rotation spectra are generally in good agreement with the observed frequency dependence of the profile. The values of the phase offsets between the three sinusoidal curves
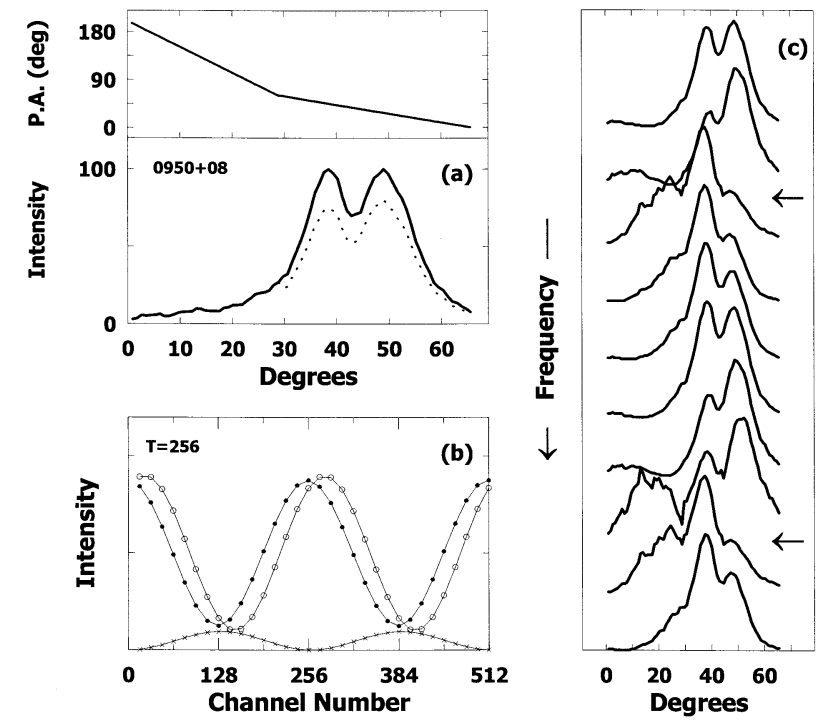

Fig. 6. A numerical study of polarization effects. a) The model profile adopted shows average profile in total intensity (solid), the linearly polarized component (dotted), and the position angle of polarization (upper panel). b) The computed Faraday rotation spectra at the longitudes of the pulse peaks of the three components: $14^{\circ}$ (crosses), $38^{\circ}$ (filled circles) and $49^{\circ}$ (open circles). c) The computed profile shapes for individual channels. All the profiles are normalized to unity. A transition state between the first and second dominant components of the main pulse is marked by an arrow.

are identical in both plots. The values of the amplitudes are slightly different. The amplitudes of the observed sinusoidal curves for the two components of the main pulse are about half that shown in Fig. 6b. Possibly, the procedure of removing the effects of scintillations, which was mentioned in Sect. 5, could partially decrease the amplitude of the wave, unrelated to scintillations. The amplitude of the third component ahead of the main pulse is 4 times greater than that plotted in Fig. $6 \mathrm{~b}$. Possibly, at $112 \mathrm{MHz}$ this component is stronger than it was assumed in our model. We do not know the true profile in total intensity at $112 \mathrm{MHz}$ and therefore the assumed ratios of amplitudes in the various profile components are approximate.

The corresponding profile shapes computed for individual channels are given in Fig. 6c. Comparison with Fig. 1 shows that the computed profiles are in good agreement with the observed ones. We clearly see the noticeable feature which characterizes the observed modulation of the pulse profiles. This is a transition state between the first and second dominant component of the main pulse. This state has a lower $\mathrm{S} / \mathrm{N}$ ratio and shows the presence of the well-resolved third component ahead of the main pulse. The well-resolved third component does not exist. The transformation of the unresolved component of the pulse profile to the well-resolved one is a result of polarization effects. The simulation of the transition state was successful when the degree of linear polarization taken was very high, more than $80 \%$. Hence, we conclude that the observed profile changes are a result of the Faraday rotation effect. 


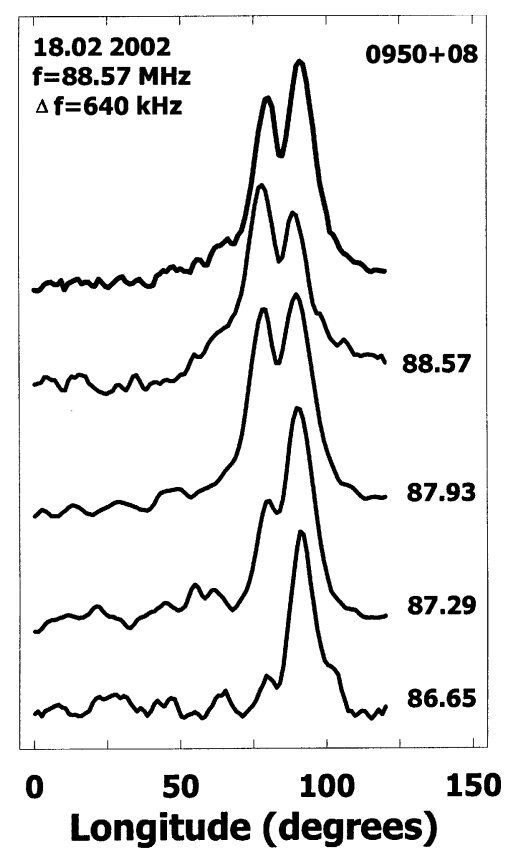

Fig. 7. The pulse profile changes at the frequency of $88.57 \mathrm{MHz}$. The upper profile is a sum of 3700 pulsar periods over a total $2.56-\mathrm{MHz}$ band. The lower profiles are that over four $640-\mathrm{kHz}$ bands. The sample interval is 1.2 of longitude. All the profiles are normalized to unity.

\section{Narrowband changes of the average profile at lower frequencies of 88,62 and $41 \mathrm{MHz}$}

Low-frequency observations of the pulsar were carried out to examine sinusoidal modulation of the pulse profile in the wide frequency range and to estimate the polarization effects corresponding to our observations. As mentioned before, the observed effects at $112 \mathrm{MHz}$ are about 3 times stronger than those predicted by the interstellar Faraday rotation.

Figure 7 displays the average pulse profile at $88.57 \mathrm{MHz}$. The profile exhibits a well-resolved double shape with a separation between the components of about $11^{\circ}$. As we can see from this plot, the profile shape varies markedly across the bandpass and these changes are similar to the changes observed at the higher frequency of $112 \mathrm{MHz}$. The transition state between the first and second dominant components was not recorded during this observation, but one can guess its presence. The frequency interval of the shape changes can be estimated to be about $3 \mathrm{MHz}$.

A similar picture of the narrowband changes of the pulse profile is observed at $62.15 \mathrm{MHz}$ as is shown in Fig. 8 . Here the profile is obtained by addition of 2200 pulse periods within eight $320-\mathrm{kHz}$ bands. The average profile has two well-resolved components with a separation of $15^{\circ}$. Obviously, the profile shape varies across the bandpass and the frequency structure of the profile is similar to that observed at higher frequencies of 88 and $112 \mathrm{MHz}$. A noticeable feature of the shape changes is the presence of two transition states between the first and second dominant components, which are clearly visible in the plot. The frequency interval of the profile changes of approximately $1.2 \mathrm{MHz}$.

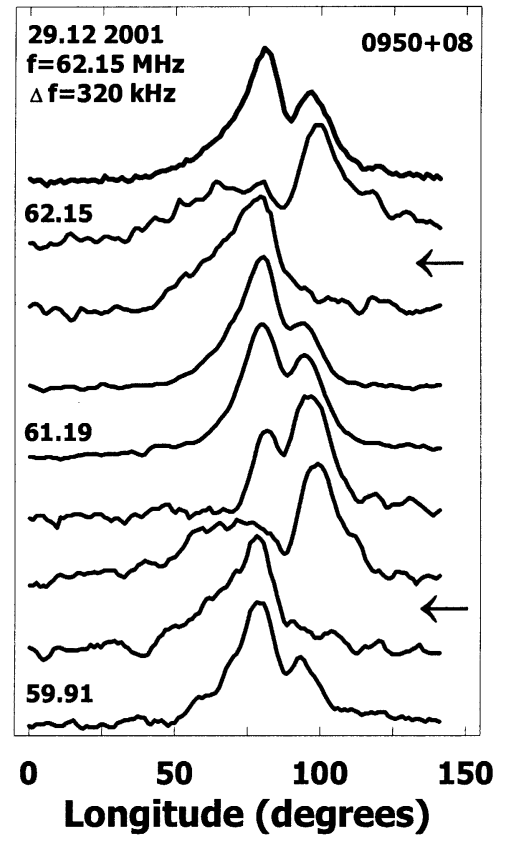

Fig. 8. The pulse profile changes at the frequency of $62.15 \mathrm{MHz}$. The upper profile is a sum of 2200 pulsar periods over a total $2.56-\mathrm{MHz}$ band. The lower profiles are that over eight $320-\mathrm{kHz}$ bands. A transition state between the first and second dominant components is marked by an arrow. The sample interval is 1.2 of longitude. All the profiles are normalized to unity.

Average profiles of the pulsar at $41.07 \mathrm{MHz}$ do not show a frequency structure similar to that observed at higher frequencies. The profiles have a lower $\mathrm{S} / \mathrm{N}$ ratio and much lower time resolution of $3 \mathrm{~ms}$, caused by the $7-\mathrm{ms}$ pulse broadening due to the dispersion effect in one $20-\mathrm{kHz}$ band. The profiles would be expected to be modulated by the interstellar Faraday rotation with the frequency scale of the order of $900 \mathrm{kHz}$. To find periodicities in the amplitude variation of the pulse profile over the bandpass of $2.56 \mathrm{MHz}$, the power spectrum was computed with the use of Fourier transform. For this procedure, we used strong individual pulses. It is well known that individual pulses are often more highly polarized than average profiles. The results are given in Fig. 9 for three different epochs. For each observation two realizations of the power spectrum are presented.

There is a distinct spectral feature at around $0.005 \mathrm{kHz}^{-1}$, which is clearly visible in the spectra for the three observations. This feature corresponds to the periodicity of approximately $200 \mathrm{kHz}$. To state with certainty the presence of this spectral feature we averaged the spectra together and presented the resulting spectrum in the lower panel of Fig. 9. As is seen, the spectral feature at $0.005 \mathrm{kHz}^{-1}$ dominates over the noisy fluctuations. This is a strong indication of the presence of sinusoidal modulation of the pulse profile at frequency of $41 \mathrm{MHz}$, too.

In this section we analyzed the narrowband profile changes in the low-frequency range from 41 to $88 \mathrm{MHz}$ and concluded that the shape changes are similar to those observed at $112 \mathrm{MHz}$. The scale of the observed profile changes is frequency dependent and varies from $3-9 \mathrm{MHz}$ at $112 \mathrm{MHz}$ to $0.2 \mathrm{MHz}$ at $41 \mathrm{MHz}$. 


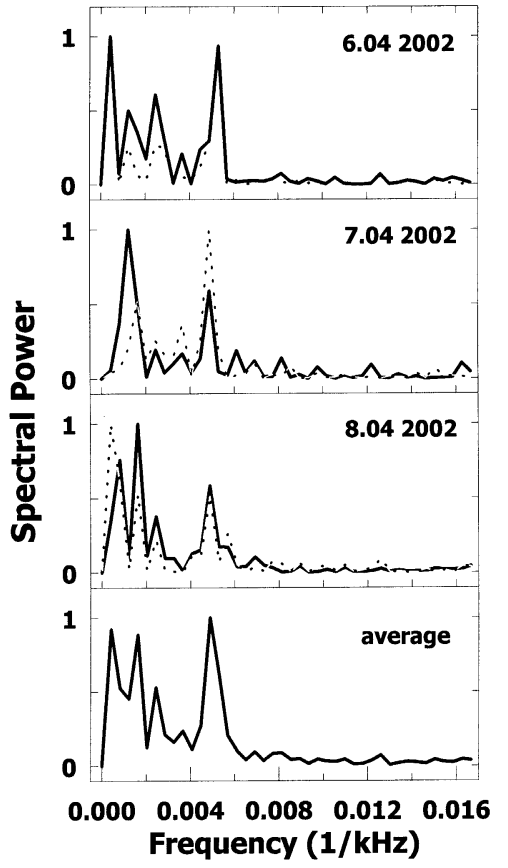

Fig. 9. The power spectra of the amplitude variations of individual pulses for PSR B0950+08 at $41 \mathrm{MHz}$ for three different observations. The spectral feature at around $0.005 \mathrm{kHz}^{-1}$ is well visible in the spectra for all the three observations. In the average spectrum shown in the lower panel this feature dominates over the noisy fluctuations.

\section{Timing residuals for PSR B0950+08}

The pulsar catalog (Taylor et al. 1993) quotes the period and the period derivative obtained by Gullahorn \& Rankin (1978) at the epoch of 1972.5. Errors in these parameters alone lead to ambiguities in the counts of the pulse number for the 1996-2001 observing session. Our measurements of the average profiles were used to improve the timing parameters for PSR B0950+08.

We analyzed the arrival times for only those average profiles whose shape looks double. They were chosen out of the full data set obtained at 102.5 and $112 \mathrm{MHz}$ between 1996 and 2001. All good-quality double profiles were summed to produce an average profile with a high $\mathrm{S} / \mathrm{N}$ ratio which was used as a template. The topocentric arrival times were calculated by cross-correlating the observed profiles with this template. Timing parameters were determined using the TEMPO software package $^{1}$ and the JPL DE200 ephemeris. The topocentric arrival times were referred to the solar system barycenter using a position and a proper motion given by Fomalont et al. (1992) and Brisken et al. (2000), respectively. A second-order polynomial describing spin-down pulsar behavior was fitted to the barycentric arrival times to obtain residuals from a timing model. Residuals were used to compute differential corrections to the initial parameter values.

The best-fitting values of the period and the period derivative are presented in Table 2. A large scatter of timing data relative to the spin-down pulsar model has not allowed us to improve the astrometric parameters. Figure 10 displays the

\footnotetext{
${ }^{1}$ http://www.atnf.csiro.au/research/pulsar/timing/ tempo
}

Table 2. Measured timing parameters for PSR B0950+08.

\begin{tabular}{ll}
\hline \hline Parameter & Value $^{a}$ \\
\hline Period, $P(\mathrm{~s})$ & $0.253065240711(4)$ \\
Period derivative, $\dot{P}\left(10^{-15} \mathrm{~s} \mathrm{~s}^{-1}\right)$ & $0.23028(5)$ \\
Epoch of period (MJD) & 50190.7360 \\
\hline
\end{tabular}

${ }^{a}$ The errors are given in units of the last quoted digit.

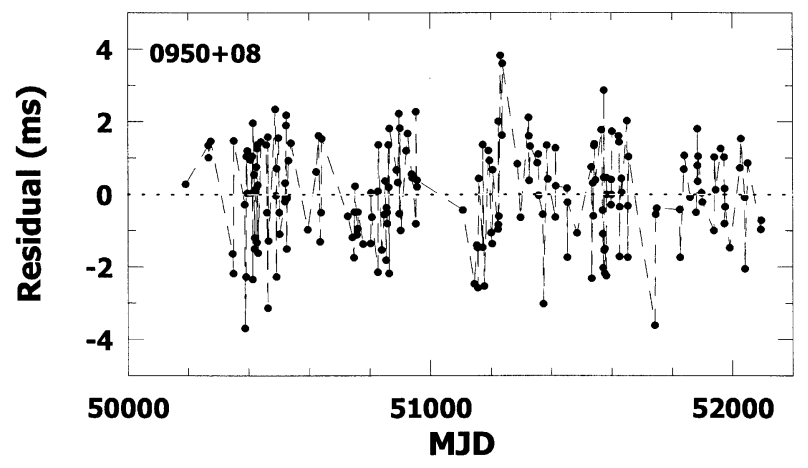

Fig. 10. Post-fit timing residuals for PSR B0950+08 between 1996 April and 2001 July.

timing residuals from the pulsar relative to their best-fit spindown model during the period 1996-2001. The rms timing residual after the fit was of the order of $1300 \mu$ s with a timing error of about $90 \mu \mathrm{s}$. At higher frequencies, above $400 \mathrm{MHz}$, where the pulsar exhibits a single pulse profile, the rms timing residual of PSR B0950+08 is much less and is estimated at $200 \mu$ s with a timing uncertainty of the order of 90-200 $\mu$ s in a 4-7 year span of data (Gullahorn \& Rankin 1978; Helfand et al. 1980). Apparently, the increase of the rms residual observed at $112 \mathrm{MHz}$ is due to the profile shape changes which cause fluctuations of the pulse arrival times and make an additional noise contribution to the timing residual.

\section{Discussion}

We have found that the average pulse profile for PSR B0950+08 observed with a single linear polarization is frequency variable all over the range $41-112 \mathrm{MHz}$. The narrowband structure of the pulse shape is associated with sinusoidal modulation of the pulsar emission. This process is frequency dependent. According to our measurements, the interval of modulation at different observing frequencies is about $3-9 \mathrm{MHz}$ at $111.87 \mathrm{MHz}, 3-4 \mathrm{MHz}$ at $88.57 \mathrm{MHz}$, $1-1.4 \mathrm{MHz}$ at $62.15 \mathrm{MHz}$ and $0.19-0.21 \mathrm{MHz}$ at $41.07 \mathrm{MHz}$. Hence, the dependence of the modulation interval on frequency is well described by a power low with index close to 3 in the range 41-112 MHz. A similar frequency structure should be observed in the presence of the Faraday rotation effect. The presence of Faraday rotation is also confirmed by a numerical simulation of the polarization effects for the pulse profiles at $112 \mathrm{MHz}$. 
The frequency interval of Faraday rotation of the plane of polarization of linear polarized emission $\Delta F_{\pi}$ at the observing frequency $v$ is determined by rotation in the position angle $\theta$ through $\pi$ and is calculated from

$\Delta F_{\pi}=\frac{\pi v^{3}}{2 R M c^{2}}=17.48 v^{3} / R M$,

where $\Delta F_{\pi}$ is in $\mathrm{MHz}, v$ is in hundreds of $\mathrm{MHz}$, the rotation measure $R M$ is in $\mathrm{rad} \mathrm{m}^{-2}$ and $c$ is the light velocity.

Using relation (5), we can estimate the rotation measure $R M$ corresponding to our measurements. For the mean values of $\Delta F_{\pi}$ equal to $6,3,1.2$ and $0.21 \mathrm{MHz}$ at the frequencies of $111.87,88.57,62.15$ and $41.07 \mathrm{MHz}$ respectively, the rotation measure $R M$ will be approximately $4 \mathrm{rad} \mathrm{m}^{-2}$. Taking into account variability of $\Delta F_{\pi}$ from one observation to another, we derive the values of $R M$ in the range 3-6 $\mathrm{rad} \mathrm{m}^{-2}$. The derived values of $R M$ are very large compared to the rotation measure $R M=1.35 \mathrm{rad} \mathrm{m}^{-2}$ measured for PSR B0950+08 at frequencies above $400 \mathrm{MHz}$ (Hamilton \& Lyne 1987; Taylor et al. 1993).

Thus, we have shown that the observed narrowband profile changes of the pulsar B0950+08 are a result of the Faraday rotation effect and could be explained as an artifact of observations of linearly polarized pulsar emission with a linearly polarized antenna. We have obtained a new value of the rotation measure corresponding to this effect, $R M \approx 4 \mathrm{rad} \mathrm{m}^{-2}$. This leads us to conclude that the published value of $R M$ is incorrect and is actually 3 times greater.

Acknowledgements. We wish to thank the staff of PRAO for assistance in the observations. This work was supported by the Russian
Foundation For Basic Research (project No. 00-02-17447). The authors are grateful to the referee for numerous helpful comments and suggestions.

\section{References}

Brisken, W. F., Benson, J. M., Beasley, A. J., et al. 2000, ApJ, 541, 959

Fomalont, E. B., Goss, W. M., Lyne, A. G., Manchester, R. N., \& Justtanont, K. 1992, MNRAS, 258, 497

Gullahorn, G. E., \& Rankin, J. M. 1978, AJ, 83, 1219

Hamilton, P. A., \& Lyne, A. G. 1987, MNRAS, 224, 1073

Hankins, T. H., \& Cordes, J. M. 1981, ApJ, 249, 241

Hankins, T. H., Izvekova, V. A., Malofeev, V. M., et al. 1991, ApJ, 373, L17

Helfand, D. J., Manchester, R. N., \& Taylor, J. H. 1975, ApJ, 198, 661

Helfand, D. J., Taylor, J. H., Backus, P. R., \& Cordes, J. M. 1980, ApJ, 237, 206

Kuzmin, A. D., Izvekova, V. A., Shitov, Yu. P., et al. 1998, A\&AS, 127,355

Lyne, A. G., \& Rickett, B. J. 1968, Nature, 218, 326

Lyne, A. G., Smith, F. G., \& Graham, D. A. 1971, MNRAS, 153, 337

Rathnasree, N., \& Rankin, J. M. 1995, ApJ, 452, 814

Schwarz, U. J., \& Morris, D. 1971, Astrophys. Lett., 7, 185

Shitov, Yu. P. 1971, AZh, 48, 638

Shitov, Yu. P. 1972, AZh, 49, 470

Smirnova, T. V., \& Shabanova, T. V. 1992, SvA, 36, 628

Suleymanova, S. A. 1989, Linear polarization of the average pulses of pulsars at 102.5, 60 and $40 \mathrm{MHz}$, in Pulsars, ed. A. D. Kuzmin, Proc. Lebedev Physical Institute, 196, 55

Taylor, J. H., Manchester, R. N., \& Lyne, A. G. 1993, ApJS, 88, 529

Udaltsov, V. A., \& Zlobin, V. N. 1974, A\&A, 37, 21

Vitkevich, V. V., \& Shitov, Yu. P. 1970, Nature, 226, 1235 